\title{
Cross-Modal Characterization for Quantifying Fracturing Fluid Effects on Organic-Rich Source Rocks
}

\author{
Weichang $\mathrm{Li}^{1}$, Bitao Lai ${ }^{1}$ and Feng Liang ${ }^{1}$ \\ ${ }^{1}$ Aramco Research Center - Houston, Aramco Services Company
}

During hydraulic fracturing and/or water injection, reservoir rock is invaded by massive amount of high pressure stimulation fluids. This may create additional micro-fractures and cause changes in porosity, permeability and mechanical properties of the rock formation [1][2]. To further quantify and understand these effects, this paper provides a cross-modal characterization of property variations in source rock samples when subject to fluid tests in a lab setting. By analytically evaluating the elemental composition changes associated with the material alteration, we aim to establish correlations between mineralogical/geochemical effects and changes in rock morphology. Specifically, morphological and compositional characterizations are obtained from Scanning electron microscope (SEM) and Energy Dispersive X-ray Spectroscopy (EDS) measurements respectively at each stage of the fluid test, targeting several overlapping areas on the same set of source rock samples. Variations and correlations are then quantified through image preprocessing, co-registration, cross-modal consistency evaluation and similarity feature ranking. Two organic rich source rock samples, one from Saudi Arabia and the other from the Eagle Ford shale in North America, which is an analogue to the Saudi sample, have been used in this study. The treatment fluids include a synthetic seawater and a slick water prepared by hydrating $0.5 \mathrm{~mL}$ of DP/EM 5015 (30\% active) in 1 liter of deionized water then followed by addition of 20 grams of $\mathrm{KCl}$.

Near surface morphology is based on the high-resolution secondary electron imaging (SEI) measurements. Figure 1 shows the SEI images of a rock sample patch containing one tight pocket of organic materials. The difference between Figure 1(a) and (b) indicates a rather mild level of dissolution as well as some sporadic deposits during seawater soaking. However, Figure 1(c) suggests significantly more severe material dissolution associated with DP/EM treatment. To quantify this type of near-surface change, we co-register these SEI images and evaluate the morphological variations based on the difference derived from the co-registered images. Figure 2 (a) and (b) show the co-registered SEI images for the same sample, before and after seawater and DP/EM treatment, respectively. The registration in this stage is among different images of the same modality. Thus it is relatively straightforward after taking into account the potential variations in SEI dynamic ranges and scanning parameters before and after the fluid test. EDS elemental maps provide the basis for our compositional analysis. Figure 3 shows the set of elemental maps for the same sample as in Figure 1 (c). For each mapped element, the pair of images before and after every fluid test stage are first normalized using histogram equalization to account for dynamic range differences, and then co-registered to evaluate variations in the elemental composition which, similar to the SEI image analysis, is of the same modality.

In order to connect morphological change with compositional variations, we then combine the information from both modalities by co-registering [3] and fusing the obtained SEI difference image with the elemental map variation images. This is done for each mapped element of interest. Based on the co-registration information, a set of similarity and consistency measures are calculated across modality and ranked over all the maps associated with the different elements. The subset of element(s) showing the highest level of consistent similarity are then identified as the most strongly correlated with the morphological alteration captured by the SEM. This provides a bridge between morphological change and compositional variations in the source rock samples subject to the fluid effects. As an example, 
Figure 4 shows over the course of the second stage fluid test, the changes in the calcite (Ca) map (a), the SEI difference image (b), and the co-registered and fused image of both (c). Calcite was subsequently identified as the major element dissolved by DP/EM 5015 fluid. This result was further confirmed by Inductively Coupled Plasma (ICP) analysis of the sample.

References:

[1] Akrad, OM. et al, Proceedings of SPE Annual Technical Conference \& Exhibition (2011) p. 146658.

[2] Lai, B. et al, Proceedings of American Rock Mechanics Association (ARMA) (2016) p.16-180

[3] Cao, T. et al, International Workshop on Biomedical Image Registration, (2012) p. 298-306.

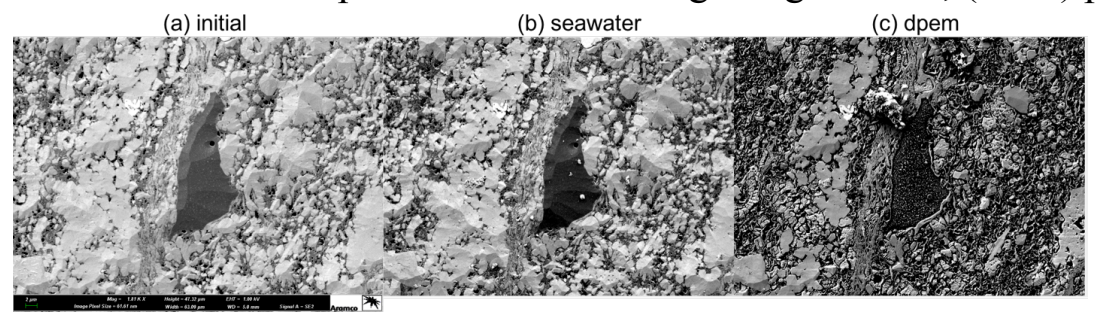

Figure 1. SEM image of (a) the initial sample (b) treated with seawater and (c) treated with DP/EM 5015

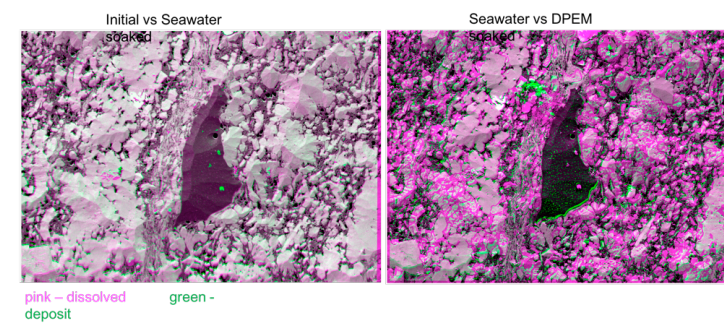

Figure 2. Topological variations induced by (a) seawater soaking (b) DP/EM 5015 fluid treatment

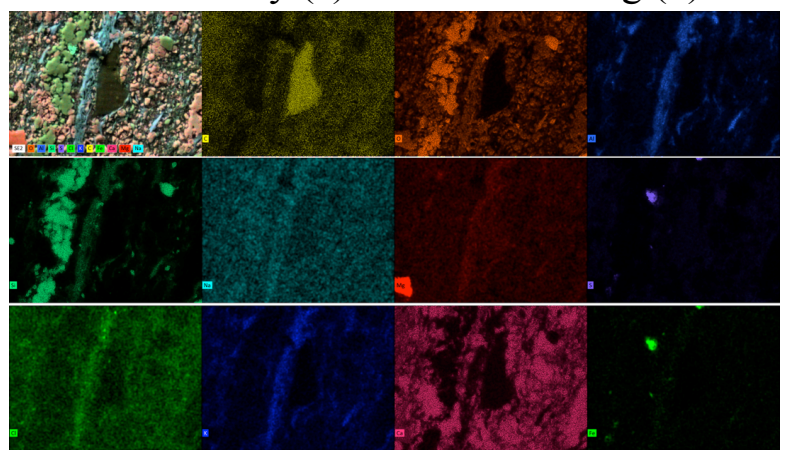

Figure 3. EDS Elemental maps of the DP/EM soaked sample, same as in Figure 1(c)

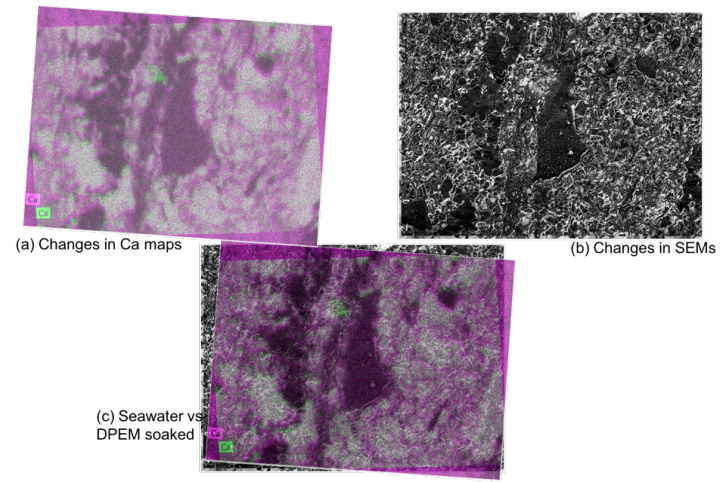

Figure 4. Consistency between morphological variations in SEM and Elemental (Ca) loss, due to DP/EM 5015 treatment 